\title{
Discovery, development, and clinical application of sugammadex sodium, a selective relaxant binding agent
}

\author{
Mark Welliver ${ }^{1,3}$ \\ John McDonough ${ }^{1,3}$ \\ Nicholas Kalynych 1,2,3 \\ Robert Redfern ${ }^{3}$ \\ 'University of North Florida, Nurse \\ Anesthetist Program, Jacksonville, \\ Florida, USA; ${ }^{2}$ Shands Jacksonville, \\ Perioperative Services, Jacksonville, \\ Florida, USA; ${ }^{3}$ University of Florida, \\ Department of Anesthesiology, \\ Jacksonville, Florida, USA
}

\begin{abstract}
Neuromuscular blockade, induced by neuromuscular blocking agents, has allowed prescribed immobility, improved surgical exposure, optimal airway management conditions, and facilitated mechanical ventilation. However, termination of the effects of neuromuscular blocking agents has, until now, remained limited. A novel cyclodextrin encapsulation process offers improved termination of the paralytic effects of aminosteroidal non-depolarizing neuromuscular blocking agents. Sugammadex sodium is the first in a new class of drug called selective relaxant binding agents. Currently, in clinical trials, sugammadex, a modified gamma cyclodextrin, has shown consistent and rapid termination of neuromuscular blockade with few side effects. The pharmacology of cyclodextrins in general and sugammadex in particular, together with the results of current clinical research are reviewed. The ability of sugammadex to terminate the action of neuromuscular blocking agents by direct encapsulation is compared to the indirect competitive antagonism of their effects by cholinesterase inhibitors. Also discussed are the clinical implications that extend beyond fast, effective reversal, including numerous potential peri-operative benefits.
\end{abstract}

Keywords: modified cyclodextrin, selective relaxant binding agent (SRBA), sugammadex, encapsulation, muscle relaxants, neuromuscular blockade reversal

\section{Introduction}

Since the introduction of curare, neuromuscular blocking agents (NMBAs) have allowed us to immobilize patients and improve medical and surgical interventions (Bohm 1912; Griffith and Johnson 1942). The synthesis and development of new NMBAs have produced agents that are close to ideal. Unfortunately, NMBA reversal agents have not been improved upon. The cholinesterase inhibitor drugs first used to reverse curare currently remain the standard for reversing all NMBAs. The indirect action of cholinesterase inhibitors is limited in its ability to reverse neuromuscular blockade, and is associated with numerous side effects. A new class of drugs, selective relaxant binding agents (SRBAs), has the potential to overcome the limitations of cholinesterase inhibitors.

The first SRBA to be introduced is sugammadex (sugammadex sodium, generic, Organon International, a part of Schering-Plough Corporation, Oss, The Netherlands), a modified gamma cyclodextrin (CD) with the chemical formula $\mathrm{C}_{72} \mathrm{H}_{104} \mathrm{Na}_{8} \mathrm{O}_{48} \mathrm{~S}_{8}$ currently in phase III clinical trials. Unlike the cholinesterase inhibitors, which indirectly and competitively antagonize NMBAs by increasing acetylcholine (ACh), sugammadex acts by directly encapsulating, binding, and inactivating NMBAs (Figure 1).

\section{Conventional neuromuscular blockade reversal}

Cholinesterase inhibitors act indirectly against the effects of NMBAs by inactivating the enzyme acetylcholinesterase (AChE) which is responsible for the breakdown 


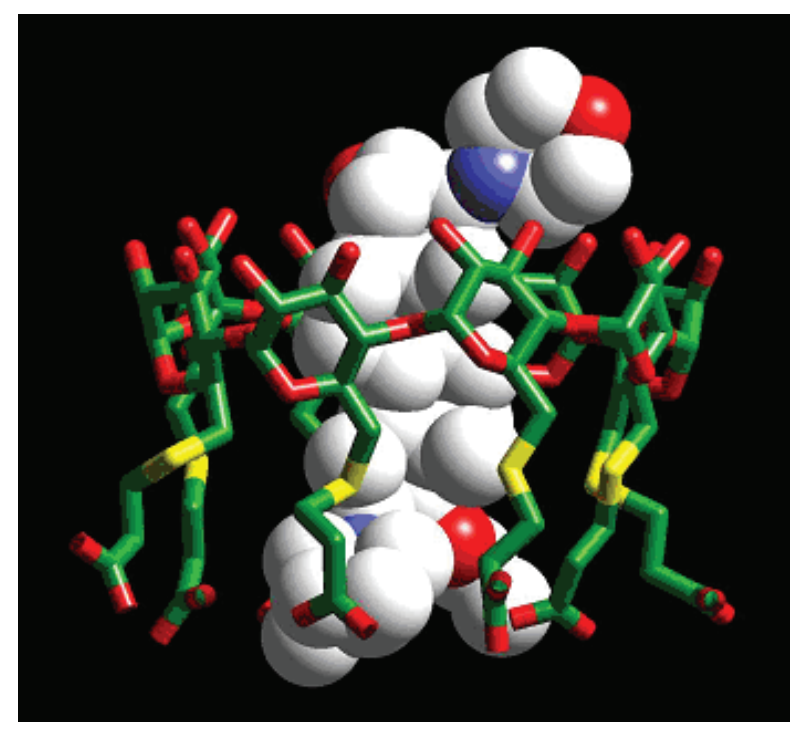

Figure I Crystal spectroscopy image of sugammadex encapsulating a rocuronium molecule. Reprinted with permission from Zhang M-Q. 2003. Drug-specific cyclodextrins: The future of rapid neuromuscular block reversal? Drugs Future, 28:347-54. Copyright $(\bigodot$ 2003 Prous Scientific and Organon USA, a part of Schering-Plough Corporation.

of ACh. Acetylcholine levels then increase dramatically, displacing the NMBA molecules from the nicotinic receptors. Thus, NMBAs are not inactivated or broken down by cholinesterase inhibitors. They are only displaced from the site of action, the nicotinic receptor. The two cholinesterase inhibitors most often used in clinical practice are edrophonium and neostigmine, which form reversible, non-covalent attachments to the anionic site or esteratic site on the AChE molecule. Their duration of action is 60 minutes or less. AChE activity returns to normal after the detachment and metabolism of cholinesterase inhibitors. The temporary increase in $\mathrm{ACh}$ then returns to normal levels. NMBA molecules still present after normalization of ACh levels may successfully compete for the nicotinic receptor and re-exert their neuromuscular blocking effects. Re-currence of paralysis (recurarization) is usually only a risk with long-acting NMBAs, although it has been reported with intermediate duration NMBAs (Singh et al 1982; Baillard et al 2000; Debaene et al 2003). Similarly, incomplete displacement (partial reversal) of NMBAs by cholinesterase inhibitors may result in residual paralysis (Hayes et al 2001; Appelboam et al 2003; Kirkegaard et al 2002; Murphy et al 2005; Murphy 2006).

\section{Residual paralysis}

The risks of residual paralysis include: dysphagia, hypoventilation, weakened hypoxic drive, impaired coughing, compromised pharyngeal and laryngeal function and pulmonary complications (Berg et al 1997; Eriksson
1999; Tramer and Fuchs-Buder 1999; Eikermann et al 2006). The primary concern in patients with residual paralysis is airway protection and adequate ventilation. Atelectesis may occur intra-operatively due to patient position, positive pressure ventilation, and hypoventilation, and is compounded in the post-operative period by any degree of residual paralysis. The residual presence of volatile anesthetics, benzodiazepines, and narcotics in the tissue compartments contribute to post-operative hypoventilation. Hypoventilation coupled with residual paralysis places a patient in less than optimal circumstances (Eriksson 1999).

Another drawback of cholinesterease inhibitors is their effects on ACh levels are not limited to the nicotinic junction. Generalized increases in ACh throughout the body causes pronounce side effects. Parasympathetic effects predominate when $\mathrm{ACh}$ is increased, leading to cardiovascular, pulmonary, gastrointestinal, and neurological sequelae. Side effects of cholinesterase inhibitors include: bradycardia, bronchospasm, increased airway secretions, nausea, vomiting, increased peristalsis, increased urination, muscle cramps/spasms, miosis, vision disturbances, and convulsions (Neostigmine Package Insert 2002). Anti-cholinergic drugs are therefore given concomitantly with cholinesterase inhibitors to attenuate their parasympathetic effects. These anti-cholinergic drugs are partially effective in this respect but exert their own side effects including: cardiac arrhythmias, tachycardia, QT prolongation, nausea, vomiting, constipation, urinary retention, severe allergic reactions, dry mouth, mydriasis, and confusion (Glycopyrrolate Package Insert 2002).

In contrast, a new concept of NMBA inactivation by SRBA encapsulation rather than competitive antagonism has been shown to be fast, effective, and safe for the reversal of neuromuscular blockade in clinical trials to date with few side effects. This encapsulation process is enabled by the unique characteristics of CDs.

\section{Cyclodextrin characteristics}

Natural CDs are found in nature wherever starch sources, bacteria, and appropriate environmental conditions exist. The glucopyranose units of amylose starch are enzymatically restructured by bacteria such as Bacillus macerans, creating the natural CDs (Szejtli 2004). The natural CDs consist of rings of six, seven, or eight glucopyranose units named alpha, beta, and gamma, respectively (Figure 2). The glucopyranose units are attached by alpha 1-4 linkages in a circular arrangement. This arrangement creates a truncated cone that points the hydroxyl groups outward along the rims 

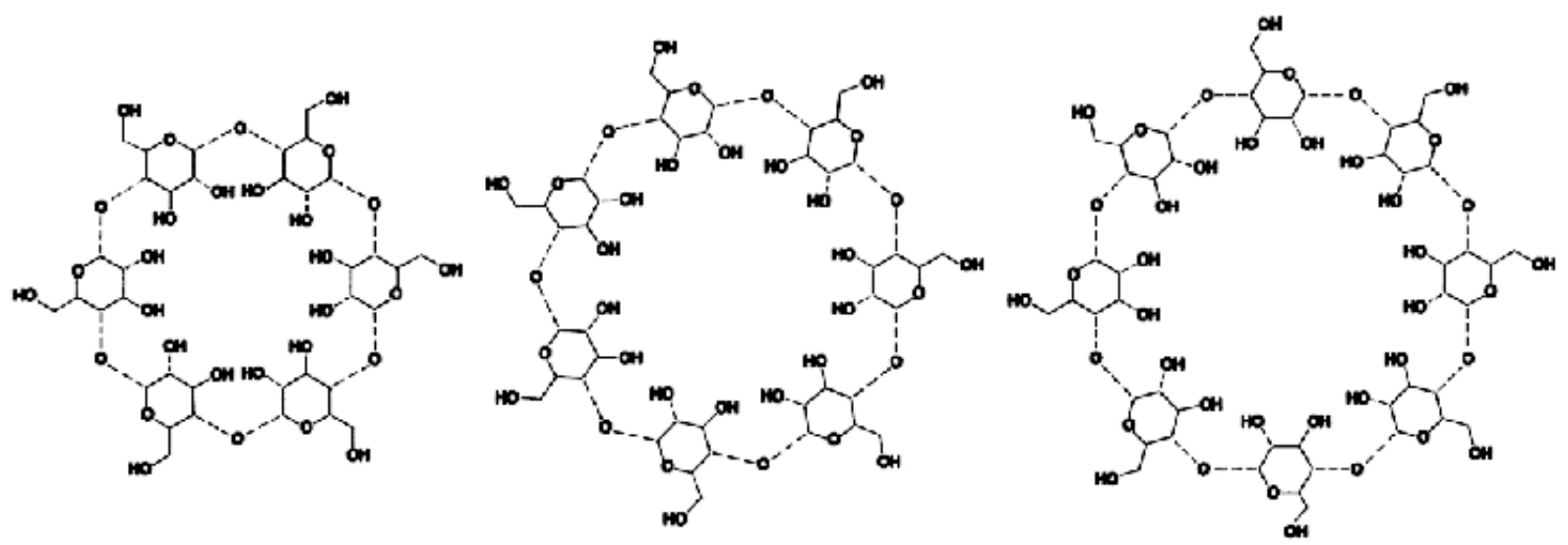

Figure 2 Naturally occurring cyclodextrins. From left to right: alpha, beta, and gamma. Sites of modification for the creation of sugammadex are the peripheral 6th carbon hydroxyl groups on the gamma cyclodextrin. Reproduced with permission from Welliver M. 2007. Update for nurse anesthetists. Part 3. Cyclodextrin introduction to anesthesia practice: form, function, and application. AANA J, 75:289-96. Copyright@ 2007 American Association of Nurse Anesthetists.

while directing the alpha 1-4 linkages inward (Figure 3). The narrow opening is called the primary face, and the larger opening is called the secondary face. The negatively charged hydroxyl groups lining the primary and secondary faces are responsible for the water solubility of these molecules. The interior of the $\mathrm{CD}$ is lined by the carbon atoms and alpha 1-4 linkages creating a lipophilic cavity. The steric nature of CDs creates a water soluble molecule that possesses a cavity capable of surrounding and binding a lipophilic molecule. The main pharmacologic benefit extracted from this structural arrangement is encapsulation of appropriately sized lipophilic

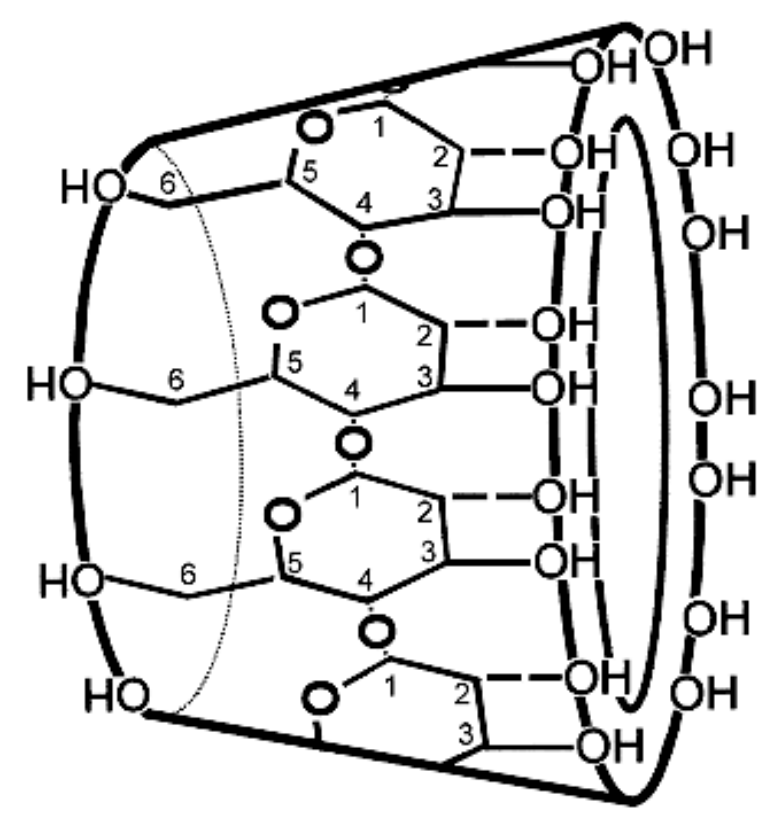

Figure 3 Structural arrangement of glucopyranose units in a gamma CD, showing primary and secondary rim hydroxyl groups. Reproduced with permission from Welliver M. 2007. Update for nurse anesthetists. Part 3. Cyclodextrin introduction to anesthesia practice: form, function, and application. AANA J, 75:289-96. Copyright ( 2007 American Association of Nurse Anesthetists. molecules or drugs and the promotion of aqueous solubility (Welliver 2007).

Historically, CDs have been considered excipients (inert adjuncts) lacking pharmacologic activity but useful for improving the formulation of other active compounds (Thompson and Chaubal 2002; Loftsson et al 2005). The two key characteristics of CDs - lipophilic molecule encapsulation and aqueous solubility - have multiple beneficial applications. For example, CD encapsulation of lipophilic drugs, which are difficult to solubilize, allows their dissolution in water, which is biologically better tolerated than the organic solvents currently used, such as benzyl alcohol and propylene glycol (MacPherson 2001). The pharmacologic benefits of CD solubilization have been explored and applied successfully with many drug formulations throughout the world (Challa et al 2005). The solubilization characteristics of CDs, coupled with biological tolerance, make them desirable replacements for other organic solvents.

Although CDs possess aqueous solubility and an affinity for lipophilic molecules, the relative potency of these characteristics varies between different CDs. Close size approximation of lipophilic molecules to their corresponding $\mathrm{CD}$ is necessary to allow non-covalent thermodynamic interactions to occur promoting the formation of an inclusion complex. The approximate cavity sizes of the natural CDs are: gamma $(0.8 \mathrm{~nm})>$ beta $(0.6 \mathrm{~nm})$ $>$ alpha $(0.5 \mathrm{~nm})$ (Welliver 2007). Once inside a CD cavity, thermodynamic attractions - including electrostatic interaction, van der Waals interaction, hydrophobic interaction, hydrogen bonding, release of conformational strain, exclusion of cavitybound high-energy water, and charge-transfer interaction - may contribute to the formation of the inclusion complex (Liu and Guo 2002). An inclusion complex, also known as a host-guest assembly, is the newly formed molecular entity of a $\mathrm{CD}$ and 
its encapsulated lipophilic molecule. Modification of the natural CDs allows improvement of their aqueous solubility and thermodynamic attractions for a particular guest molecule. The sites available for $\mathrm{CD}$ modification are on the 2nd, 3rd, and 6th carbon atoms of the glucopyranose sub-units (Figure 3). Numerous substituent atoms or groups may be placed on any or all of these sites improving a CD's affinity for a particular molecule (Szente and Szejtli 2002). The ability to improve upon the base characteristics of natural CDs has led to the discovery of a $\mathrm{CD}$ that promises the unique drug effect of NMBA encapsulation with resultant termination of the paralytic effect.

\section{Discovery of the modified cyclodextrin sugammadex}

The impetus for discovery of the modified gamma CD sugammadex was the desire to improve the solubility of the NMBA rocuronium (Zhang 2003). Rocuronium is a non-depolarizing NMBA with a steroidal backbone that is commercially prepared in an aqueous solution adjusted to a pH of 4.0 (Rocuronium package insert 2007). Researchers at Organon Laboratories (New House, UK) wanted to solubilize rocuronium without the acidic mannitol phosphate buffer solvent used in the current formulation. A different rocuronium formulation was considered desireable in order to eliminate the acidic $\mathrm{pH}$ as an extraneous variable in in vitro experiments of the smooth muscle effects of rocuronium (Bom and Hope 2007). The exploration of suitable solvents led to CDs, which have been known and used to solubilize lipophilic molecules for over 100 years (Szetjtli 2004).

Understanding the potential to achieve $\mathrm{CD}$ encapsulation specific for rocuronium, researchers explored many modifications of the alpha, beta, and gamma CDs (Adam et al 2002; Tarver et al 2002) The candidate CD chosen to solubilize rocuronium was the modified gamma CD Org 25969 (sugammadex). Sugammadex is a modified gamma $\mathrm{CD}$ with every 6th carbon hydroxyl group substituted with a thioether linked to a carboxyl group (Figure 4). The thioether linkages extend the cavity length allowing complete encapsulation of the rocuronium molecule while increasing the area over which thermodynamic attractions can occur (Bom et al 2002a; Bom et al 2002b; Zhang 2003) A secondary benefit provided by the negatively charged carboxyl groups is an increased aqueous solubility of the sugammadex molecule (Zhang 2003) (Figure 5). X-ray crystallography has shown very close size compatibility between sugammadex and the rocuronium molecule (Bom et al 2002a) Furthermore, it was found to be one of the most stable complexes of a $\mathrm{CD}$ and its guest molecule, with an association constant $\left(\mathrm{K}_{\mathrm{a}}\right)$ of $1.8 \times 10^{7} \mathrm{M}^{-1}$ (Bom et al

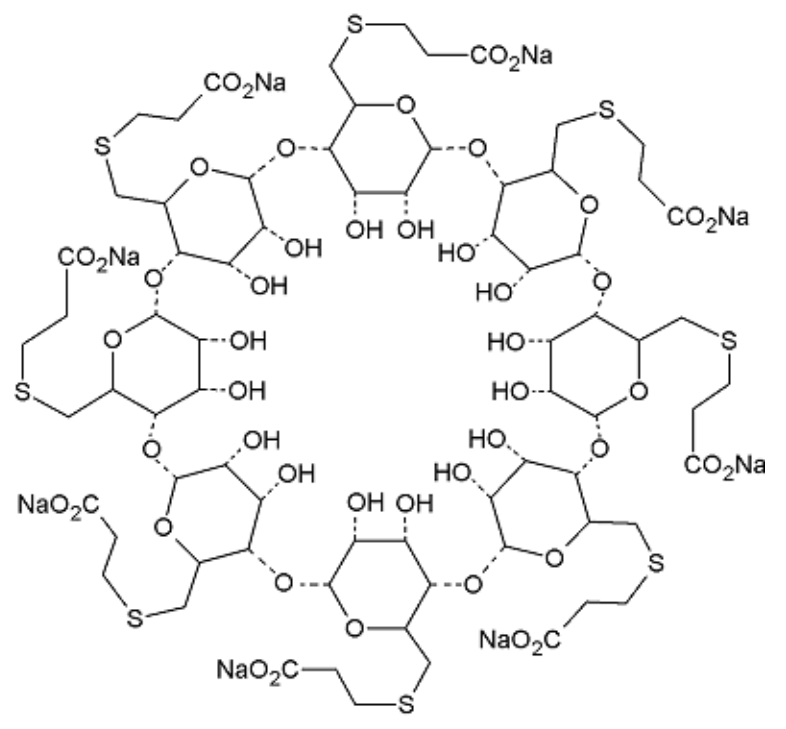

Figure 4 Sugammadex structure. Reproduced with permission from Welliver M. 2007. Update for nurse anesthetists. Part 3. Cyclodextrin introduction to anesthesia practice: form, function, and application.AANA J, 75:289-96. Copyright(C 2007 American Association of Nurse Anesthetists.

2002a) Many unrelated drug/CD complexes studied previously have averaged $\mathrm{K}_{\mathrm{a}} \mathrm{s}$ of $1 \times 10^{1} \mathrm{M}^{-1}$ to $2 \times 10^{4} \mathrm{M}^{-1}$ (Challa et al 2005). The discovery of sugammadex's extremely high binding affinity for rocuronium changed the research focus from in vitro drug solubilization to the novel concept of in vivo drug extraction (Bom et al 2007). The new concept of encapsulation termination of the effects of NMBAs was born.

The high lipophilic binding affinity of sugammadex raised concerns about potential undesirable binding to endogenous and exogenous steroidal compounds. These concerns were addressed during early studies. Zhang found over 40 lipophilic, steroidal, and non steroidal drugs typically given during an anesthetic case had affinities with sugammadex that ranged from 120-700 times less than that of rocuronium (Zhang 2003). These drugs include induction agents (propofol, thiopenthane), narcotics (fentanyl, remifentanyl), antibiotics (vancomycin, gentamycin), bronchodilators (salbutamol, aminophyline), cardiovascular drugs (atropine, digoxin, ephedrine, phentolamine, verapamil), and steroids (cortisone, hydrocortisone) (Zhang 2003). Clinical studies also showed no evidence of interaction with or alteration of the volatile inhalation anesthetic sevoflurane or the intravenous anesthetic propofol (Vanacker et al 2007). Elsewhere, it was demonstrated that propanolol and isoprenaline did not modify the action of sugammadex (Bom and Hope 2007). Thus, sugammadex has high selectivity for the aminosteroidal NMBAs.

\section{Mechanism of action}

Sugammadex is unique in its application, since CDs have been used to encapsulate lipophilic molecules in vitro in 


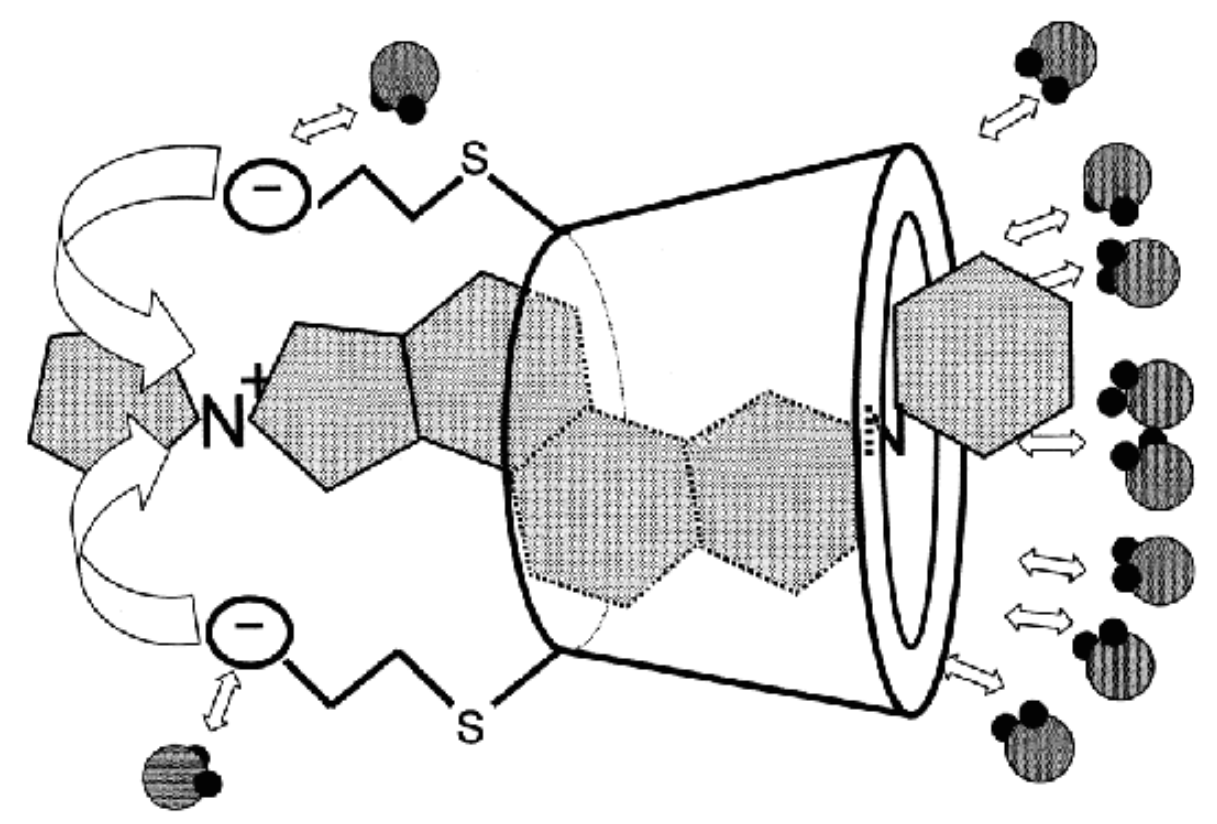

Figure 5 Aqueous inclusion complex of sugammadex-rocuronium.

order to increase solubilization for improved drug delivery. Sugammadex is the first CD created to perform its own drug effect. The process of sugammadex reversal of NMBA effects can be broken down into two component parts, direct molecular encapsulation (Figure 6) and mass extraction of NMBAs from the nicotinic junction into the plasma (Figure 7). Sugammadex is administered intravenously and remains in the plasma without diffusing or being transported to other compartments. In the plasma, sugammadex encapsulates and non-covalently binds amonosteroidal NMBAs with a one-to-one ratio. The specifics of this encapsulation process have been the focus of early studies. Using microcrystalography, Bom determined that all four steroidal rings of the rocuronium molecule lie in close approximation to the lipophilic cavity of the sugammadex molecule, with the quaternary containing ring (D) surrounded by the carboxyl groups (Bom et al 2002a). It was believed that thermodynamic interactions occurring between the sugammadex cavity and the steroidal backbone of the rocuronium molecule, along with the carboxyl groups attractions for rocuronium's tertiary ammonium accounted for its total high binding affinity (Adam et al 2002; Zhang 2003).

The plasma encapsulation of NMBA molecules prevents their diffusion into the peripheral compartment for attachment to nicotinic ACh receptors. In addition, NMBA molecules already attached to extracellular nicotinic receptors, causing neuromuscular blockade are rapidly drawn into the plasma and encapsulated. This removal of NMBA molecules from nicotinic $\mathrm{ACh}$ receptors occurs because of the concentration gradient created by sugammadex binding of the NMBAs in the plasma (Epemolu et al 2003). That is, the shift to a low concentration of unbound NMBA molecules in the plasma causes the higher concentration of extracellular NMBA molecules to detach from the $\mathrm{ACh}$ receptors and diffuse into the plasma. Once back in the plasma, these NMBA molecules are quickly encapsulated and bound by sugammadex. This process occurs rapidly, restoring motor function.

The two-fold process of one-to-one encapsulation/ binding in the plasma and the concentration gradientmediated extraction of NMBAs from the nicotinic ACh receptors results in fast and effective termination of neuromuscular blockade by sugammadex. The ability of sugammadex to encapsulate and reverse the effects of aminosteroidal NMBAs varies between agents. Sugammadex reverses rocuronium greater than vecuronium and much greater than pancuronium (Alvarez-Gomez et al 2007; Decoopman et al 2007; Duvaldestin et al 2007).

\section{Preclinical studies}

An overview of the research shows the development and progression of a safe and efficacious reversal drug that is poised to enable dramatic improvements in the management of neuromuscular blockade. Animal studies confirmed the high affinity of sugammadex for both rocuronium and vecuronium and, to a lesser extent, pancuronium with the ability to terminate NMBA effects under multiple conditions. (Welliver 2006). Mason successfully reversed rocuronium- and vecuronium-induced 


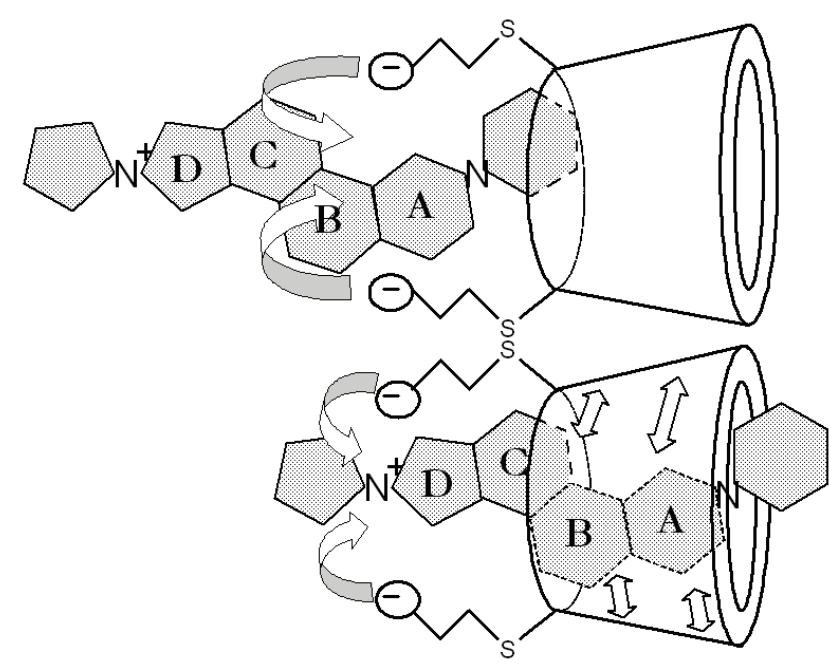

Figure 6 Encapsulation process: Sugammadex carboxyl groups interact with steroidal rings $A, B, C$, and $D$, drawing the aminosteroidal NMBA molecule into the cavity where additional non-covalent attractions hold the molecule securely in place.

neuromuscular blockade in guinea pigs with sugammadex $1 \mathrm{mg} / \mathrm{kg}$ in less than 1 minute (Mason and Bom 2001). Profound neuromuscular block induced by rocuronium 500 $\mu \mathrm{g} / \mathrm{kg}$ in Rhesus monkeys was successfully performed by de Boer using $2.5 \mathrm{mg} / \mathrm{kg}$ sugammadex (de Boer et al 2006).

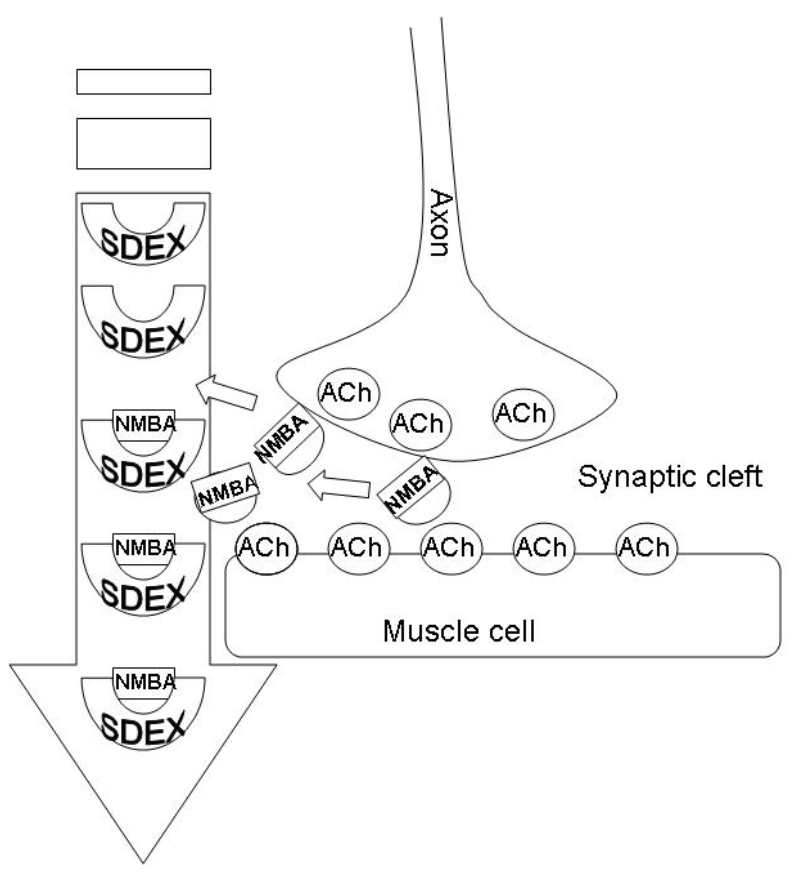

Figure 7 Sugammadex encapsulates aminosteroidal NMBA molecules in the plasma causing a concentration gradient that favors the extraction of additional rocuronium molecules from the nicotinic junction into the plasma. Reproduced with permission from Harris AM, Welliver M, Redfern R, et al. 2007. Orthopaedic surgery implications of a novel encapsulation process that improves neuromuscular blockade and reversal. The Internet Journal of Orthopedic Surgery, 7(2). Copyright@) ISPUB.com.
Sugammadex reversal of rocuronium was unaffected by $\mathrm{pH}$ changes in guinea pigs or renal impairment in cats (Bom et al 2002c; Bom et al 2003).

\section{Clinical studies Phase I studies}

Human studies of sugammadex were first performed by Gijsenbergh in 29 healthy male volunteers. All were anesthetized and had neuromuscular blockade induced by rocuronium. Either placebo or sugammadex, in doses ranging from $0.1-8.0 \mathrm{mg} / \mathrm{kg}$, were given, and both time and degree of reversal were recorded. Sugammadex $8.0 \mathrm{mg} / \mathrm{kg}$ reversed rocuronium-induced blockade in 1 minute, compared to 52 minutes with placebo. The investigators concluded that effective and safe reversal of rocuronium-induced neuromuscular blockade could be achieved with sugammadex. (Gijsenbergh et al 2005).

\section{Phase II studies}

A phase II study also demonstrated safe reversal of rocuronium-induced neuromuscular blockade of 2 hours or greater duration (Shields et al 2006). In this study of 30 anesthetized patients age $>18$ years, ASA class I-III, sugammadex was administered at doses of $0.5-6.0 \mathrm{mg} / \mathrm{kg}$. At doses of $1.0 \mathrm{mg} / \mathrm{kg}$ and above, sugammadex effectively reversed the effects of rocuronium in a dose-dependant fashion within 4 minutes (Shields et al 2006). Two studies in 87 patients age 18-87 years, ASA class I-III, explored the use of sugammadex for reversal of high-dose rocuronium (1.0 and 1.2 $\mathrm{mg} / \mathrm{kg}$ ) (Khunl-Brady et al 2005; Rex et al 2005). Sugammadex $(2-16 \mathrm{mg} / \mathrm{kg})$ was administered at 3 and 15 minutes after rocuronium administration. The average time to reversal (TOF 0.9) with $8.0 \mathrm{mg} / \mathrm{kg}$ sugammadex was $<3$ minutes at both time intervals with both doses of rocuronium. It was concluded that there was a clear dose-response relationship in terms of time to recovery with sugammadex (Khunl-Brady et al 2005; Rex et al 2005) de Boer also found profound rocuronium-induced $(1.2 \mathrm{mg} / \mathrm{kg})$ neuromuscular blockade given with propofol and opioid anesthesia of greater than 90 minutes duration was effectively reversed by sugammadex in a dose dependent manner in 45 patients aged 18-64 years, ASA class I-II (de Boer et al 2007).

Studies of sugammadex for the reversal of shallow vecuronium-induced neuromuscular blockade have also shown a dose-dependent time to full reversal. Puhringer reversed both vecuronium- and rocuronium-induced neuromuscular blockade in 100 patients, age 20-65 years, ASA class I-III (Puhringer et al 2007). Sugammadex doses of 
$2.0 \mathrm{mg} / \mathrm{kg}$ for rocuronium and $4.0 \mathrm{mg} / \mathrm{kg}$ for vecuronium effectively reversed shallow neuromuscular blockade (TOF 2/4) in 3 minutes or less. In another study, profound vecuronium-induced neuromuscular blockade (PTC 1-2) was effectively reversed by sugammadex $4.0 \mathrm{mg} / \mathrm{kg}$ in an average time of 4.5 minutes (Lemmens et al 2007). Dosedependent reversal of both rocuronium- and vecuroniuminduced profound neuromuscular blockade by sugammadex was also observed by Duvaldestin in a study of 102 patients, age 21-64 years, ASA class I-III (Duvaldestin et al 2007). Sugammadex $2.0 \mathrm{mg} / \mathrm{kg}$ reversed profound rocuroniuminduced neuromuscular blockade (PTC 1-2) in 3.5 minutes or less, while a greater dose of $4.0 \mathrm{mg} / \mathrm{kg}$ was required to reverse vecuronium-induced profound neuromuscular blockade within the same amount of time.

In addition to the intermediate duration NMBAs rocuronium and vecuronium, the effects of the long acting NMBA pancuronium have also been successfully reversed by sugammadex (Decoopman et al 2007). In this study of 20 patients, age 20-81 years, ASA class I-III, shallow (TOF 2/4) pancuronium-induced neuromuscular blockade was effectively reversed in less than 3 minutes with a sugammadex dose of $4.0 \mathrm{mg} / \mathrm{kg}$. The authors concluded that sugammadex had a "good safety profile". Interestingly, a significant doseresponse relationship was not shown in this study. The effectiveness of sugammadex for the reversal of pancuronium, in addition to rocuronium and vecuronium, is significant in light of the limitations of cholinesterase inhibitors.

The efficacy of sugammadex for the reversal of neuromuscular blockade has been compared to that of the cholinesterase inhibitor reversal drugs neostigmine and edrophonium. In one study the speed and effectiveness of sugammadex $(4.0 \mathrm{mg} / \mathrm{kg})$ for reversal of profound vecuronium-induced neuromuscular blockade (PTC 1-2) was compared to that of neostigmine (70 $\mu \mathrm{g} / \mathrm{kg}$ ) (Lemmens et al 2007). With neostigmine, the average reversal time to a TOF ratio of 0.9 was 66.2 minutes compared to an average time of 4.5 minutes with sugammadex. Another study comparing sugammadex $(4.0 \mathrm{mg} / \mathrm{kg})$ and neostigmine $(70 \mu \mathrm{g} / \mathrm{kg})$ for reversal of profound rocuronium-induced neuromuscular blockade (PTC 1-2) also observed a much faster return to a TOF ratio of 0.9 with sugammadex ( 2.9 minutes) than neostigmine (50.4 minutes) (Jones et al 2007).

The results of these phase II clinical studies have consistently demonstrated that sugammadex achieves fast and effective reversal of various degrees and durations of rocuronium- and vecuronium-induced neuromuscular blockade. Shallow pancuronium-induced neuromuscular blockade has also been effectively reversed (Deccopman et al 2007). Comparisons with cholinesterase inhibitors have shown faster and more thorough reversal accomplished with sugammadex with an improved safety profile (Lemmens et al 2007; Jones et al 2007).

\section{Phase III studies}

In a phase III study in 100 patients, Alvarez-Gomez reported faster reversal of vecuronium using sugammadex $2.0 \mathrm{mg} / \mathrm{kg}$ compared to neostigmine $50 \mu \mathrm{g} / \mathrm{kg}$ (Alvarez-Gomez et al 2007). The median time for reversal of shallow (TOF 2/4) vecuronium-induced neuromuscular blockade to a recovery of 0.9 TOF ratio was 2.1 minutes for sugammadex and 18.9 minutes for neostigmine. In another study, reversal of moderately profound rocuronium-induced neuromuscular blockade was also found to be faster with sugammadex $4.0 \mathrm{mg} / \mathrm{kg}$ (average 1.78 minutes) verses edrophoniumatropine (average 5.51 minutes) and neostigmine-glycopyrrolate (average 17.4 minutes) (Sacan et al 2007). All patients reversed with sugammadex achieved full reversal to a TOF ratio of 0.9 . Only $10 \%$ of the edrophonium-atropine reversed patients and $25 \%$ of the neostigmine-glycopyrrolate reversed patients achieved full reversal within 30 minutes. Interestingly, all patients were able to perform a 5-second head lift after all reversal drugs. The sugammadex group experienced significantly lower incidences of increased heart rate and dry mouth.

Additional comparison of sugammadex to conventional cholinesterase inhibitor reversal therapy has demonstrated a faster time to full reversal of rocuronium- and vecuroniuminduced neuromuscular blockade by sugammadex. In a study with 98 patients age $>18$ years, ASA class I-III, sugammadex was compared to neostigmine reversal (Blobner et al 2007). Sugammadex $2.0 \mathrm{mg} / \mathrm{kg}$ or neostigmine $50 \mu \mathrm{g} / \mathrm{kg}$ along with glycopyrrolate $10 \mathrm{mcg} / \mathrm{kg}$ was administered at a TOF $2 / 4$. The mean time to full reversal was 1.4 minutes for sugammadex and 17.6 min for neostigmine. Flockton compared sugammadex reversal of rocuronium effects to neostigmine reversal of the benzyl isoquinoline NMBA cis-atracurium. Sugammadex $2.0 \mathrm{mg} / \mathrm{kg}$ was delivered at TOF $2 / 4$ after rocuronium $0.6 \mathrm{mg} / \mathrm{kg}$ in 34 patients. Neostigmine $50 \mathrm{mcg} / \mathrm{kg}$ along with glycopyrrolate $10 \mu \mathrm{g} / \mathrm{kg}$ was delivered at TOF $2 / 4$ after cis-atracurium $0.15 \mathrm{mg} / \mathrm{kg}$ in 39 patients. The mean time to full reversal was 1.51 minutes for sugammadex compared to 2.85 minutes for neostigmine (Flockton et al 2007).

Phase III clinical trials have also explored the effects of sugammadex in pediatric and elderly patients, and in patients with renal, pulmonary, and cardiac disease. A study by Plaud 
investigated rocuronium-induced $(0.6 \mathrm{mg} / \mathrm{kg})$ neuromuscular blockade in 8 infants (age 28 days to 23 months), 24 children (age 2-11 years), 31 adolescents (age 12-17 years), and 28 adults (age 18-65 years) anesthetized with propofol and opioids or caudal anesthesia (Plaud et al 2007). When shallow neuromuscular block was confirmed by TOF 2/4, sugammadex doses of $0.5-4.0 \mathrm{mg} / \mathrm{kg}$ were administered. A dose-dependent time to TOF ratio 0.9 was demonstrated in all groups except the infant group due to the low number of subjects. The median time to recovery was less than 3 minutes with sugammadex doses of $1.0,2.0$, and $4.0 \mathrm{mg} / \mathrm{kg}$ in all groups.

The efficacy of sugammadex for the reversal of shallow rocuronium-induced neuromuscular blockade (TOF 2/4) was studied in 48 adult patients (age 18-64 years), 62 elderly (age 65-74 years), and 40 old elderly patients (age $>75$ years) (McDonagh 2007). All patients received rocuronium $0.6 \mathrm{mg} / \mathrm{kg}$ followed by sugammadex $2 \mathrm{mg} / \mathrm{kg}$ at a TOF 2/4. The mean time to recovery of the TOF ratio to 0.9 was 2.3 minutes in the adult group, 2.6 minutes in the elderly group, and 3.6 minutes in the old elderly group. The estimated time to TOF 0.9 in all patients over 65 years of age was 2.9 minutes versus 2.3 minutes in the adult group, a statistically significant difference $(\mathrm{p}=0.022)$.

The effectiveness of sugammadex for the reversal of rocuronium-induced neuromuscular blockade was assessed in 15 patients with impaired renal function (creatinine clearance $<30 \mathrm{~mL} / \mathrm{min}$ ) and 15 patients with normal renal function (creatinine clearance $>80 \mathrm{~mL} / \mathrm{min}$ ) (Staals et al 2007). All patients were anesthetized with propofol and opioids, and had neuromuscular blockade induced with rocuronium $0.6 \mathrm{mg} / \mathrm{kg}$. At the reappearance of TOF $2 / 4$, sugammadex $2.0 \mathrm{mg} / \mathrm{kg}$ was administered. The mean time to recovery of TOF ratio to 0.9 was 2.0 minutes in the renal impaired group and 1.7 minutes in group with normal renal function. The authors reported no signs of recurarization or adverse effects, and concluded that sugammadex rapidly and completely reverses rocuronium-induced neuromuscular blockade in patients with normal or impaired renal function.

Patients with pulmonary disease were studied to examine the possible respiratory effects of sugammadex. Seventyseven patients 18 years or older, ASA class II-III, with a known history or diagnosis of pulmonary disease were anesthetized and administered rocuronium $(0.6 \mathrm{mg} / \mathrm{kg})$ (Amao et al 2007). At the end of surgery and reappearance of TOF 2/4, sugammadex 2.0 or $4.0 \mathrm{mg} / \mathrm{kg}$ was administered. The mean time to recovery of the TOF ratio to 0.9 was 1.8 minutes in the sugammadex $4.0 \mathrm{mg} / \mathrm{kg}$ group, and 2.1 minutes for the $2.0 \mathrm{mg} / \mathrm{kg}$ group. Two serious episodes of bronchospasm occurred that were considered possibly related to sugammadex, both of which were in the $4.0 \mathrm{mg} / \mathrm{kg}$ dose group. Both patients had a history of asthma. No alterations in respiratory rate or recurarization were observed in any patients. The authors concluded sugammadex was well tolerated and effective for the reversal of rocuronium-induced neuromuscular blockade in patients with pulmonary disease.

One hundred twenty-one patients with cardiac disease (NYHA class II-III, ASA class II-IV, age 36-90 years) undergoing elective non-cardiac surgery, were anesthetized and received rocuronium (Dahl et al 2007). At the reappearance of TOF $2 / 4$, sugammadex 2.0 or $4.0 \mathrm{mg} / \mathrm{kg}$ was administered. The mean time to a TOF ratio of 0.9 was found to be 1.7 minutes with sugammadex $2.0 \mathrm{mg} / \mathrm{kg}$ and 1.4 minutes with the $4.0 \mathrm{mg} / \mathrm{kg}$ dose. The authors disclosed two serious adverse events (SAEs) relating to prolongation of the QTc interval in the placebo and sugammadex groups. "Of these, one case of QTc interval prolongation in each treatment group (placebo and sugammadex group) was considered by the investigator to be possibly related to study treatment". Analysis of the mean QTc interval using the Fridericia correction (US Dept HHS 2005) was performed. Safe and effective use of sugammadex in cardiac patients was concluded. However, the general anesthetics used in this study, which may cause QTc prolongation, were not disclosed in this preliminary report.

\section{Reported adverse effects}

Few adverse effects have been attributed to sugammadex. One study reported abdominal discomfort as "definitely related" to study drug (Suy et al 2007). Movement after sugammadex was observed in several studies which may be expected with rapid restoration of motor function and lighter anesthetic levels towards the conclusion of surgery (Sorgenfrei et al 2006; de Boer et al 2007). Possible adverse effects reported in clinical trials include: erythema, alterations in taste and smell, coughing, dry mouth, tachycardia, bradycardia, pyrexia, dizziness, vomiting, hypotension, abnormal urine levels of $\mathrm{N}$-acetyl-glucosamninidase, and QT interval prolongation (Gijsenbergh et al 2005; Shields et al 2006; Sorgenfrei et al 2006; Dahl et al 2007). QT prolongation was first observed by Rex but was considered not to be related to sugammadex (Rex et al 2005). An analysis by Vanacker determined that corrected QT prolongations, which were not arrhythmogenic, were likely to be due to the anesthetics sevoflurane and propofol (Vanacker et al 2007). A study by de Kam using the criteria of the International Conference on Harmonisation 
(ICH-E14) guidelines (Shah 2005) found that sugammadex doses up to $32 \mathrm{mg} / \mathrm{kg}$ were not associated with QT/QTc prolongation (de Kam et al 2007). The authors of a study in cardiac patients also reported that no QTc prolongations were related to sugammadex (Dahl et al 2007).

Inadvertent over dosage occurred in one clinical study, when sugammadex $40 \mathrm{mg} / \mathrm{kg}$ was administered instead of $4.0 \mathrm{mg} / \mathrm{kg}$, but this was not associated with any adverse effects and effective reversal of rocuronium was reported. (Molina et al 2007) Although most disclosed adverse effects have been expressed as only "possibly related" to the study drug, conclusive determination has yet to be made. It should be noted that some phase III studies have not been completed at the time of writing, and unknown side effects may exist. The results from one comparative study suggested that "the safety profile of sugammadex was at least as good as that of neostigmine" (Jones et al 2007).

\section{Metabolism and excretion}

Sugammadex is biologically well tolerated. It does not undergo metabolism or breakdown and therefore does not affect blood sugar levels. It is excreted by the kidneys intact and has been found to increase the excretion of rocuronium molecules that it encapsulates (Sorgenfrei et al 2006). The renal excretion of sugammadex mirrors glomerular filtration rate (Gijsenbergh et al 2005) and it can be removed by dialysis (Hartman et al 2007).

\section{Clinical implications of direct acting reversal by encapsulation}

\section{Intraoperative}

The ability to reverse NMBAs directly and completely will allow the provision of continual profound neuromuscular blockade that may currently be inadvisable due to the limitations of cholinesterase inhibitor reversal agents. Multiple surgical specialties are likely to find this beneficial. With incomplete neuromuscular blockade, surgeons may encounter unnecessary difficulties. For example, those who require open access to abdominal cavities may struggle with retractors to improve visualization of structures. Others may find patient extremities resistant to manipulation.

Patient attempts at spontaneous ventilation are evidence of incomplete neuromuscular blockade. Diaphragmatic excursion represents significant return of neuromuscular function and closely correlates with motor function return of the abdominal rectus muscles. Motor function disrupts on optimal exposure and causes displacement of abdomi- nal viscera. In contrast, profound neuromuscular blockade assures no diaphragmatic movement or abdominal rectus tone, and optimizes surgical access. Consistent profound neuromuscular blockade may also allow access through smaller incisions as muscular tension and contractions are eliminated as surgical hindrances.

Laproscopic procedures are assisted by assured patient immobility, as coughing or "bucking" under anesthesia increases the risk of organ injury and interferes with video imagining. Fracture reductions and manipulation of extremities may also be conducted with greater ease and speed in the absence of muscular tone or resistance. With the availability of sugammadex, surgical procedures requiring profound neuromuscular block may be accomplished without the risks associated with incomplete reversal by cholinesterase inhibitors. That is, by means of its rapid and complete reversal of rocuronium and vecuronium, sugammadex will allow surgeons to operate with profound neuromuscular blockade up to and including wound closure.

\section{Neuromuscular monitoring}

In light of the effectiveness of SRBAs, the question of continued use of neuromuscular monitoring has been raised (Kopman 2006; Naguib 2007). Neuromuscular monitoring should be used whenever neuromuscular blocking agents are administered. In the interests of improving vigilance, and because of concerns about potentially insufficient sugammadex doses, we suggest the continued use of nerve stimulators to assess the degree of neuromuscular blockade. Eleveld described a scenario in which initial recovery to a TOF ratio of 0.9 quickly deteriorated to $<0.3$ due to an insufficient dose of sugammadex $(0.5 \mathrm{mg} / \mathrm{kg})$ delivered for the reversal of profound rocuronium-induced neuromuscular blockade in a dose-finding study (Eleveld et al 2007). Consistent and effective dosages based on degree of blockade will be determined, however, individual variability and under-dosing may allow recurarization such as that reported by Eleveld. Clinical assessment and neuromuscular monitoring should remain a mainstay of anesthesia practice (Eriksson 2003).

\section{Summary}

An opportunity is emerging that will allow profound neuromuscular blockade during surgery with full and immediate reversal upon conclusion. Reversal of neuromuscular blockade with the novel agent sugammadex is likely to increase the speed of patient recovery from anesthesia and improve time to discharge. Delivery of a safer, more effective drug 
for reversal of NMBA will also provide additional benefits. For example, the assurance of full reversal of neuromuscular blockade will allow improved conditions for surgery and, possibly, improved patient recovery. The rapid return of neuromuscular function achieved by sugammadex may also facilitate rapid sequence intubation using the nondepolarizing NMBA rocuronium, and play a role in the management of the "cannot intubate, cannot ventilate scenario. Rescue of residual paralysis resulting from incomplete reversal by cholinesterase inhibitors may also be performed quickly. Differential diagnosis of low level residual paralysis versus other compromising factors may also be achieved with sugammadex.

Patient outcome studies comparing conventional NMBA reversal with cholinesterase inhibitors to reversal by means of CD encapsulation are needed to determine the potential benefits of sugammadex beyond effective and immediate NMBA reversal. Complete control of neuromuscular blockade with immediate reversibility will allow anesthetists to offer surgeons optimal conditions that are not always achieved at present. A full appreciation of SRBA pharmacology will become known with continued clinical study, but, the successful outcomes of studies to date appear to be heralding in a new era in neuromuscular blockade management.

\section{References}

Adam JM, Bennett DJ, Bom A, et al. 2002. Cyclodextrin-derived host molecules as reversal agents for the neuromuscular blocker rocuronium bromide: synthesis and structure-activity relationships. J Med Chem, 45:1806-16.

Alvarez-Gomez JA, Wattwil M, Vanacker B, et al. 2007. Reversal of vecuronium-induced shallow neuromuscular blockade is significantly faster with sugammadex compared with neostigmine. Eur J Anaesthesiol, 24(Suppl 39):124-5.

Amao R, Zornow MH, Cowan MR, et al. 2007. Sugammadex safely reverses rocuronium-induced blockade in patients with pulmonary disease. Anesthesiology, 107:A1582.

Appelboam R, Mulder R, Saddler J. 2003. Atracurium associated with post operative residual curarization. Correspondence. Br J Anaesthesiol, 90:523-8.

Baillard C, Gehan G, Reboul-Marty J, et al. 2000. Residual curarization in the recovery room after vecuronium. Br J of Anaesthesiol, 84:394-5.

Berg H, Roed J, Viby-Mogenson J, et al. 1997. Residual neuromuscular block is a risk factor for postoperative pulmonary complications. A prospective, randomized, and blinded study of postoperative pulmonary complications after atracurium, vecuronium, and pancuronium. Acta Anaesthesiol Scand, 41:1095-103.

Blobner M, Eriksson L, Scholz J, et al. 2007. Sugammadex 92.0 mg/kg) significantly faster reverses shallow rocuronium-induced neuromuscular blockade compared with neostigmine (50 mcg/kg). Eur J Anaesthsiol, 24(Suppl 39):125.

Bohm R. 1912. Ueber die Verbindung der Lokalanasthesie mit der Narkose, uber hohe Extraduralanasthesie und epidurale Injektion anastesierender Losenungen bei tabischen Magenkrisen. Beitrage zur Klinischen Chirurgie. 80:168-89.
Bom A, Bradley M, Cameron K, et al. 2002a. A novel concept of reversing neuromuscular block: chemical encapsulation of rocuronium bromide by a cyclodextrin-based synthetic host. Agnew Chem Int Ed, 41:266-70.

Bom A, Clark JK, Palin R. 2002b. New approaches to reversal of neuromuscular block. Curr Opin Drug Discov Develop, 5:793-800.

Bom A, Epemolu O, Hope F, et al. 2007. Selective relaxant binding agent for reversal of neuromuscular blockade. Curr Opin Pharmacol, 7:298-302.

Bom A, Hope F. 2007. Propanolol and isprenaline do not modify the sugammadex-induced fast recovery from neuromuscular blockade. Eur J Anaesthesiol, 24:111-12.

Bom A, Mason R, McIndewar I. 2002c. Org 25969 causes rapid reversal of rocuronium-induced neuromuscular block, independent of acid-base status. Anesthesiology, 96:A-1009.

Bom A, Van Egmond J, Hope F, et al. 2003. Rapid reversal of rocuroniuminduced neuromuscular block by Org 25969 is independent of renal perfusion. Anesthesiology, 99:A-1158.

Challa R, Ahula A, Ali J, et al. 2005. Cyclodextrins in drug delivery: an updated review. AAPS Pharma Sci Tech, 6:329-57.

Debaene B, Plaud B, Dilly MP, et al. 2003. Residual paralysis in the PACU after a single intubating dose of nondepolarizing muscle relaxant with an intermediate duration of action. Anesthesiology, 98:1042-8.

Dahl V, Pendeville PE, Hollman MW, et al. 2007. Reversal of rocuroniuminduced blockade by sugammadex in cardiac patients. Anesthesiology, 107:A1581.

de Boer H, Driessen J, Marcus M, et al. 2007. Reversal of rocuroniuminduced $(1.2 \mathrm{mg} / \mathrm{kg})$ profound neuromuscular block by sugammadex: a multicenter, dose-finding and safety study. Anesthesiology, 107:239-44.

de Boer HD, van Egmond J, van de Pol F, et al. 2006. Reversal of profound neuromuscular blockade by sugammadex in anesthesized rhesus monkeys. Anesthesiology, 104:718-23.

Decoopman M, Cammu G, Suy K, et al. 2007. Reversal of pancuroniuminduced block by the selective relaxany binding agent sugammadex. Eur J Anaesthesiol, 24(Suppl 39):110-11.

de Kam P-J, van Kuijk J, Smeets J, et al. 2007. Single IV sugammaedx doses up to $32 \mathrm{mg} / \mathrm{kg}$ are not associated with QT/QTc prolongation. Anesthesiology, 107:A1580.

Duvaldestin P, Kuizenga K, Kjaer CC, et al. 2007. Sugammadex achieves fast recovery from profound neuromuscular blockade induced by rocuronium or vecuronium: a dose-response study. Eur J Anaesthesiol, 24(Suppl 39):123.

Eikerman M, Blobner M, Groeben H, et al. 2006. Postoperative upper airway obstruction after recovery of the train of four ratio of the adductor pollicis muscle from neuromuscular blockade. Anesth Analg, 102:937-42.

Eleveld DJ, Kuizenga K, Proost JH, et al. 2007. A temporary decrease in twitch response during reversal of rocuronium-induced muscle relaxation with a small dose of sugammadex. Anesth Analg, 104:582-4.

Epemolu O, Bom A, Hope F, et al. 2003. Reversal of neuromuscular blockade and simultaneous increase in plasma rocuronium concentration after the intravenous infusion of the novel reversal agent Org 25969. Anesthesiology, 99:632-7.

Eriksson LI. 1999. The effects of residual neuromuscular blockade and volatile anesthestics on the control of ventilation. Anesth Analg, 89:243-51.

Flockton E, Scanni E, Gomar C, et al. 2007. Sugammadex after rocuronium provides faster recovery from neuromuscular blockade than neostigmine after cisatracurium. Eur J Anaesthesiol, 24(Suppl 39):123.

Gijsenbergh F, Ramael S, Houwing N, et al. 2005. First human exposure of Org 25969. A novel agent to reverse the action of rocuronium bromide. Anesthesiology, 103:695-703.

Glycopyrrolate injection package insert. 2002. American Regent Laboratories, Inc. Sept. 2002. Shirley NY. USA.

Griffith HR, Johnson GE. 1942. The use of curare in general anesthesia. Anesthesiology, 3:418. 
Harris AM, Welliver M, Redfern R, et al. 2007. Orthopaedic surgery implications of a novel encapsulation process that improves neuromuscular blockade and reversal. The Internet Journal of Orthopedic Surgery, 7(2).

Hartmann J, Smeets JMW, deZwart MAH, et al. 2007. In-vitro dialysability of sugammadex, a selective relaxant binding agent for reversal of neuromuscular block induced by rocuronium. Accessed March 10, 2007.Available at: www.euroanesthesia.org/abstractresults/Madrid2006/f-h.php.

Hayes AH, Mirakhur RK, Breslin DS, et al. 2001. Postoperative residual block after intermediate-acting neuromuscular blocking drugs. Anesthesia, 56:312-18.

Jones RK, Caldwell JE, Brull SJ, et al. 2007. Faster reversal of profound rocuronium-induced neuromuscular blockade with sugammadex vs neostigmine. Anesthesiology, 107:A1577.

Khunl-Brady K, Rex C, Sielenkamper A, et al. 2005. Reversal of high-dose rocuronium with Org 25969. Eur J Anaesthesiol, 22(Suppl 34):121.

Kirkegaard H, Heier T, Caldwell J. 2002. Efficacy of tactile-guided reversal from cisatracurium-induced neuromuscular block. Anesthesiology, 96:45-50.

Kopman AF. 2006. Sugammadex: A revolutionary approach to neuromuscular antagonism. Anesthesiology, 104:631-3.

Lemmens HJM, El-Orbany MI, Berry J, et al. 2007. Sugammadex reverses profound vecuronium blockade more rapidly than neostigmine Anesthesiology, 107:A1578.

Loftsson T, Pekka J, Mar M, et al. 2005. Cyclodextrins in drug delivery. Expert Opin Drug Deliv, 2:1-17.

Liu L, Guo Q-X. 2002. The driving forces in the inclusion complexation of cyclodextrins. Journal of Inclusion Phenomena and Macrocyclic Chemistry, 42:1-14.

MacPherson RD. 2001. Pharmaceuticals for the anaesthetist. Anaesthesia, 56:965-79.

Mason R, Bom A. 2001. Org 25969 causes selective reversal of neuromuscular block induced by steroidal NMBs in anesthetized guinea pigs. Eur J Anaesthesiol, 18(Suppl 23):100.

McDonagh DL, Benedict PE, Kovac AL, et al. 2007. Efficacy and safety for reversal of rocuronium-induced blockade in elederly patients. Anesthesiology, 107:A1583.

Molina AL, de Boer HD, Klimekl M, et al. 2007. Reversal of rocuroniuminduced (1.2 mg kg-1) profound neuromuscular block by accidental high dose of sugammadex (40 mg kg-1). Br J Anaesth, 98:624-7.

Murphy GS. 2006. Residual neuromuscular blockade: incidence, assessment, and relevance in the postoperative period. Minerva Anesthesiol, 72:97-109.

Murphy GS, Szokol JW, Marymont JH, et al. 2005. Residual paralysis at the time of tracheal extubation. Anesth Analg, 100:1840-1845.

Naguib M. 2007. Sugammadex: Another milestone in clinical neuromuscular pharmacology. Anesth Analg, 104:575-81.

Neostigmine package insert. 2002. American Regent Laboratories, Inc. Sept. 2002. Shirley NY. USA.

Plaud B, Meretoja O, Pohl B, et al. 2007. Reversal of rocuronium-induced neuromuscular blockade with sugammadex in paediatric and adult patients. Eur J Anaesthesiol, 24(Suppl 39):124.

Puhringer F, Blaszyk M, Cammu G, et al. 2007. Sugammadex achieves fast recovery from shallow neuromuscular blockade induced by rocuronium of vecuronium: dose-response studies. Eur J Anaesthesiol, 24 (Suppl 39): 111.

Rex C, Khuenl-Brady K, Sielenkaemper, et al. 2005. Reversal of high-dose rocuronium $(1.2 \mathrm{mg} / \mathrm{kg})$ with Org 25969. Anesthesiology, A1129.

Rocuronium Bromide package insert. 2007. Organon International. Oss Netherlands. Accessed Oct 9, 2007. URL: http://www.zemuron. com/hcp/productsummary/chemistry/index.asp?C $=86765393246433$ 101852\&svarqvp2 $=0$.
Sacan O, White PF, Tufanogullari B, et al. 2007. Sugammadex reversal of rocuronium-induced neuromuscular blockade: a comparison with neostigmine-glycopyrrolate and edrophonium-atropine. Anesth Analg, 104:569-74

Shah RR. 2005. Drugs, QTc interval prolongation and final ICH E14 guideline: An important milestone with challenges ahead. Drug Saf, 28:1009-28.

Shields M, Giovannelli M, Mirakhur RK, et al. 2006. Org 25069 (sugammadex) a selective relaxant binding agent for antagonism of prolonged rocuronium-induced neuromuscular blockade. $\mathrm{Br} J$ Anaesthesiol, 96:36-43.

Singh PD, Kaur J, Sidhu GS. 1982. Assessment of post-operative residual paralysis with muscle relaxants. Indian Journal of Anaesthesia, 30:483-8.

Staals LM, Snoek MMJ, Flockton E, et al. 2007. The efficacy of sugammadex in subjects with impaired renal function. Eur $J$ Anaesthesiol, 24(Suppl 39):122-3.

Sorgenfrei IF, Norrild K, Larsen PB, et al. 2006. Reversal of rocuroniuminduced neuromuscular block by the selective relaxant binding agent sugammadex: a dose-finding and safety study. Anesthesiology, 104:667-74

Suy K, Morias K, Cammu G, et al. 2007. Effective reversal of moderate rocuronium- or vecuronium-induced neuromuscular block with sugammadex, a selective relaxant binding agent. Anesthesiology, 106:283-8.

Szente J, Szejtli J. 1999. Highly soluble cyclodextrin derivatives: chemistry, properties, and trends in development. Adv Drug Deliv Rev, 36:17-28.

Szejtli Jozsef. 2004. Past, present, and future of cyclodextrin research. Pure Appl Chem, 76:1825-45.

Tarver GJ, Grove SJ, Buchanan K, et al. 2002. 2-O-substituted cyclodextrins as reversal agents for the neuromuscular blocker rocuronium bromide. Bioorg Med Chem, 10:1819-27.

Thompson DO, Chaubal MV. 2002. Cyclodextrins (CDS) excipients by definition, drug delivery systems by function (part I: injectable applications). Drug Delivery Technology. 2(7):34-38.

Tramer MR, Fuchs-Buder T. 1999. Omitting antagonism of neuromuscular block: effect on postoperative nausea and vomiting and risk of residual paralysis. A systematic review. Br J Anaesthesiol, 82:379-86.

United States Department of Health and Human Services. 2007. Guidance for Industry. E14 clinical evaluation of QT/QTc interval prolongation and proarrythmic potential for non-antiarrythmic drugs. Oct. 2005. Accessed Sept 10, 2007. URL: http://www.fda.gov/cber/gdlns/iche14qtc.htm.

Vanacker B, Vermeyen K, Struys MRF, et al. 2005. Reversal by Org 25969 is not affected by sevoflurane when compared with propofol. Eur $J$ Anaesthesiol, 22(Suppl 34):119.

Vanacker BF, Vermeyen KM, Struys MMRF, et al. 2007. Reversal of rocuronium-induced neuromuscular block with the novel drug sugammadex is equally effective under maintenance anesthesia with propofol or sevoflurane. Anesth Analg, 104:563-8.

Welliver M. 2006. New drug sugammadex. AANA J, 74:357-63.

Welliver M. 2007. Update for nurse anesthetists. Part 3. Cyclodextrin introduction to anesthesia practice: form, function, and application. AANA J, 75:289-96.

Zhang M-Q. 2003. Drug-specific cyclodextrins: The future of rapid neuromuscular block reversal? Drugs Future, 28: 347-54. 
